\title{
Erratum to: Simulation of InGaN/GaN light-emitting diodes with patterned sapphire substrate
}

\author{
Chang Sheng Xia - Z. M. Simon Li • \\ Yang Sheng • Li Wen Cheng
}

Published online: 4 November 2014

C Springer Science+Business Media New York 2014

\section{Erratum to: Opt Quant Electron (2013) 45:605-610 DOI 10.1007/s11082-013-9675-3}

In our published manuscript, the simulated results for PSS case A in Fig. 4 were incorrect. The correct results can be seen in the following figure:

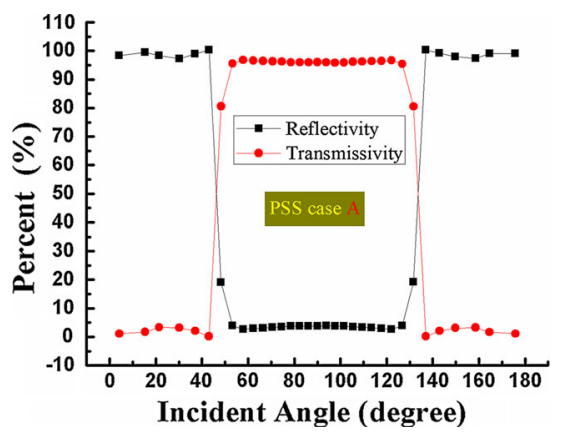

The online version of the original article can be found under doi:10.1007/s11082-013-9675-3.

C. S. Xia $(\varangle) \cdot$ Z. M. S. Li · Y. Sheng

Crosslight Software Inc. China Branch, Suite 906, Building JieDi, 2790 Zhongshan Bei Road, Shanghai 200063, China

e-mail: xiachsh@crosslight.com.cn

L. W. Cheng

National Laboratory for Infrared Physics, Shanghai Institute of Technical Physics,

Chinese Academy of Sciences, 500 Yu Tian Road, Shanghai 200083, China 